\title{
A COMPARISON PRINCIPLE FOR PARABOLIC COMPLEX MONGE-AMPĖRE EQUATIONS
}

\author{
HOANG-SON DO AND THANH CONG NGOC PHAM
}

\begin{abstract}
Dedicated to Professor Ahmed Zeriahi on the occasion of his retirement.
\end{abstract}
\begin{abstract}
In this paper, we study the Cauchy-Dirichlet problem for Parabolic complex Monge-Ampère equations on strongly pseudoconvex domains using the viscosity method. We prove a comparison principle for Parabolic complex Monge-Ampère equations and use it to study the existence and uniqueness of viscosity solution in certain cases where the sets $\{z \in \Omega: f(t, z)=0\}$ may be pairwise disjoint.
\end{abstract}

\section{Contents}

1. Introduction 1

2. Preliminaries 3

3. Some useful lemmas 6

4. Proof of Theorem 1.1 and Corollary $1.2 \quad 11$

$\begin{array}{ll}\text { Acknowledgements } & 14\end{array}$

$\begin{array}{ll}\text { References } & 14\end{array}$

\section{INTRODUCTION}

In Algebraic Geometry, the Minimal Model Program is known as a process of simplifying algebraic varieties through algebraic surgeries in birational geometry. In [14, 13], Song and Tian gave a conjectural picture to approach the Minimal Model Program via the KählerRicci flow. This approach requires a theory of weak solutions for certain degenerate parabolic complex Monge-Ampère equations.

A viscosity approach for parabolic Monge-Ampère (PMA) equations has been developed by Eyssidieux-Guedj-Zeriahi both on domains [4] and on compact Kähler manifolds $[5,6]$ (see also [2] and [15] for some generalizations). In another direction, a theory of pluripotential solutions for PMA equations has been developed in $[7,8]$. Under suitable conditions, the notions of these weak solutions are equivalent [9]. Besides having applications for the Minimal Model Program, the theories of weak solutions for PMA equations are interesting topics in themself. The aim of this paper is to study the theory of viscosity solutions for PMA equations in domains of $\mathbb{C}^{n}$.

Date: October 8, 2021

Keywords: Viscosity solutions, Parabolic Monge-Ampère equation, pluripotential theory.

The first author was supported by Vietnam Academy of Science and Technology under grant number CT0000.07/21-22. 
Let $\Omega \subset \mathbb{C}^{n}$ be a strongly pseudoconvex domain and let $T \in(0, \infty)$. We consider the following Cauchy-Dirichlet problem:

$$
\left\{\begin{array}{l}
\left(d d^{c} u\right)^{n}=e^{\partial_{t} u+F(t, z, u)} \mu(t, z) \quad \text { in } \quad \Omega_{T} \\
u=\varphi \quad \text { in } \quad[0, T) \times \partial \Omega \\
u(0, z)=u_{0}(z) \quad \text { in } \bar{\Omega}
\end{array}\right.
$$

where

- $\Omega_{T}=(0, T) \times \Omega$.

- $F(t, z, r)$ is continuous in $[0, T] \times \bar{\Omega} \times \mathbb{R}$ and non-decreasing in $r$.

- $\mu(t, z)=f(t, z) d V$, where $d V$ is the standard volume form in $\mathbb{C}^{n}$ and $f \geq 0$ is a bounded continuous function in $[0, T] \times \Omega$.

- $\varphi(t, z)$ is a continuous function in $[0, T] \times \partial \Omega$.

- $u_{0}(z)$ is continuous in $\bar{\Omega}$ and plurisubharmonic in $\Omega$ such that $u_{0}(z)=\varphi(0, z)$ in $\partial \Omega$.

In [4], Eyssidieux-Guedj-Zeriahi proved that if $\left(u_{0}, \mu(0, z)\right)$ is admissible (see Definition 2.10) and $F, f, \varphi$ do not depend on $t$ then (1) has a unique viscosity solution. In [2], this result has been extended to the case where $F, f, \varphi$ depend on $t$ and $f$ satisfies some additional conditions under which $\{z \in \Omega: f(t, z)=0\} \subset\{z \in \Omega: f(s, z)=0\}$ for $0<s<t<T$ (see [2, Theorem 4.13]). In the general case, with $f$ being merely a nonnegative, bounded, continuous function, the question about the existence and uniqueness of viscosity solution to (1) is still open.

In this paper, we prove a comparison principle for (1) and use it to study the existence and uniqueness of viscosity solution to (1) in certain cases where the sets $\{z \in \Omega$ : $f(t, z)=0\}$ may be pairwise disjoint. Specifically, we assume that $\Phi:(-1,1) \times \Omega \rightarrow \mathbb{C}^{n}$ is a continuous mapping satisfying the following conditions:

- the mapping $z \mapsto \Phi(s, z)$ is holomorphic in $\Omega$ for every $s \in(-1,1)$;

- $\Phi(0, z)=z$ for every $z \in \Omega$;

- $\partial_{t} \Phi$ is well defined and continuous on $(-1,1) \times \Omega$. In particular, $\partial_{t} \Phi$ is holomorphic on $\{0\} \times \Omega$ and, for each $U \Subset \Omega$, there exist $C_{U}>0$ and $\delta_{U}>0$ such that $\Phi(s, z) \in \Omega$ and

$$
|\Phi(s, z)-z| \leq C_{U}|s|
$$

for all $(s, z) \in\left(-\delta_{U}, \delta_{U}\right) \times U$.

Our main result is as follows:

Theorem 1.1. Suppose that the following conditions are satisfied

- for every $0<R<S<T$ and $K \Subset \Omega$, there exist $a, b>0$ such that if $\left(t_{0}, z_{0}\right) \in$ $(R, S) \times K$ and $f\left(t_{0}, z_{0}\right)=0$ then

$$
f\left(t_{0}, z\right) \leq \exp \left(b-a \frac{\left|\left\langle z-z_{0}, \partial_{t} \Phi\left(0, z_{0}\right)\right\rangle\right|}{\left|z-z_{0}\right|^{2}}\right),
$$

for every $z \in \Omega \backslash\left\{z_{0}\right\}$;

- for every $0<R<S<T, K \Subset \Omega$ and $\epsilon>0$, there exists $0<\delta<\delta_{K}$ such that

$$
(1+\epsilon) f(t, z) \geq f(t+s, \Phi(s, z)),
$$

for every $z \in K, R<t<S$ and $|s|<\delta$. 
Assume that $u$ and $v$, respectively, is a bounded viscosity subsolution and a bounded viscosity supersolution to (1). Then, for every $0<R<S<T, K \Subset \Omega$ and $\epsilon>0$, there exists $0<\delta<\delta_{K}$ such that

$$
u\left(t+s_{1}, \Phi\left(s_{1}, z\right)\right)<v\left(t+s_{2}, \Phi\left(s_{2}, z\right)\right)+\epsilon,
$$

for all $z \in K, R<t<S$ and $\max \left\{\left|s_{1}\right|,\left|s_{2}\right|\right\}<\delta$.

It is easy to see that if $f$ does not depend on $t$ then $f$ satisfies (3) and (4) with $\Phi(s, z)=z$. Some other simple examples are $(f, \Phi)=\left(g\left(t z_{0}+z\right),-s z_{0}+z\right)$ and $(f, \Phi)=$ $\left(g\left(e^{i t} z\right), e^{-i s} z\right)$, where $z_{0} \in \mathbb{C}^{n}$ and $g(z)=e^{-1 /(h(z))^{2}}$ for some Lipschitz function $h$. If $f_{1}, f_{2}$ satisfy (3) and (4) for the same $\Phi$ then $t f_{1}+(T-t) f_{2}$ satisfies (3) and (4).

We expect that Theorem 1.1 still holds without the condition (3). We need this condition for some estimates in the proof of Lemma 3.3.

By using Theorem 1.1, we obtain the following result:

Corollary 1.2. Assume that $\left(u_{0}, \mu(0, z)\right)$ is admissible (see Definition 2.10). Suppose that $\Phi$ and $f$ satisfy the conditions in Theorem 1.1. Then (1) has a unique viscosity solution.

\section{Preliminaries}

In this section, we recall some basic concepts and well-known results about viscosity sub/super-solutions. The reader can find more details in [12], [10], [11], [1], [3] and [2].

Definition 2.1. (Test functions) Let $w: \Omega_{T} \longrightarrow \mathbb{R}$ be any function defined in $\Omega_{T}$ and $\left(t_{0}, z_{0}\right) \in \Omega_{T}$ a given point. An upper test function (resp. a lower test function) for $w$ at the point $\left(t_{0}, z_{0}\right)$ is a $C^{(1,2)}$-smooth function $q$ (i.e., $\partial_{t} q, D_{z} q$ and $D_{z}^{2} q$ are continuous on on the domain of $q)$ in a neighbourhood of the point $\left(t_{0}, z_{0}\right)$ such that $w\left(t_{0}, z_{0}\right)=q\left(t_{0}, z_{0}\right)$ and $w \leq q$ (resp. $w \geq q)$ in a neighbourhood of $\left(t_{0}, z_{0}\right)$.

Definition 2.2. 1. A function $u \in U S C\left(\Omega_{T}\right)$ is said to be a (viscosity) subsolution to the parabolic complex Monge-Ampère equation

$$
\left(d d^{c} u\right)^{n}=e^{\partial_{t} u+F(t, z, u)} \mu(t, z),
$$

in $\Omega_{T}$ if for any point $\left(t_{0}, z_{0}\right) \in \Omega_{T}$ and any upper test function $q$ for $u$ at $\left(t_{0}, z_{0}\right)$, we have

$$
\left(d d^{c} q_{t_{0}}\left(z_{0}\right)\right)^{n} \geq e^{\partial_{t} q\left(t_{0}, z_{0}\right)+F\left(t_{0}, z_{0}, q\left(t_{0}, z_{0}\right)\right)} \mu\left(t_{0}, z_{0}\right) .
$$

In this case, we also say that $u$ satisfies the differential inequality

$$
\left(d d^{c} u\right)^{n} \geq e^{\partial_{t} u(t, z)+F(t, z, u(t, z))} \mu(t, z),
$$

in the viscosity sense in $\Omega_{T}$.

A function $u \in U S C([0, T) \times \bar{\Omega})$ is called a subsolution to the Cauchy-Dirichlet problem (1) if $u$ is a subsolution to (5) satisfying $u \leq \varphi$ in $[0, T) \times \partial \Omega$ and $u(0, z) \leq u_{0}(z)$ for all $z \in \Omega$.

2. A function $v \in \Omega_{T}$ is said to be a (viscosity) supersolution to the parabolic complex Monge-Ampère equation (5) in $\Omega_{T}$ if for any point $\left(t_{0}, z_{0}\right) \in \Omega_{T}$ and any lower test function $q$ for $v$ at $\left(t_{0}, z_{0}\right)$ such that $d d^{c} q_{t_{0}}\left(z_{0}\right) \geq 0$, we have

$$
\left(d d^{c} q_{t_{0}}\right)^{n}\left(z_{0}\right) \leq e^{\partial_{t} q\left(t_{0}, z_{0}\right)+F\left(t_{0}, z_{0}, q\left(t_{0}, z_{0}\right)\right)} \mu\left(t_{0}, z_{0}\right) .
$$

In this case we also say that $v$ satisfies the differential inequality

$$
\left(d d^{c} v\right)^{n} \leq e^{\partial_{t} v(t, z)+F(t, z, v(t, z))} \mu(t, z),
$$


in the viscosity sense in $\Omega_{T}$.

A function $v \in \operatorname{LSC}([0, T) \times \bar{\Omega})$ is called a supersolution to (1) if $v$ is a supersolution to (5) satisfying $v \geq \varphi$ in $[0, T) \times \partial \Omega$ and $v(0, z) \geq u_{0}(z)$ for all $z \in \Omega$.

3. A function $u$ is said to be a (viscosity) solution to (5) (respectively, (1)) if it is a subsolution and a supersolution to (5) (respectively, (1)).

Remark 2.3. a) By the same argument as in the proof of [3, Proposition 1.3], if $u$ is a subsolution to (5) and $q$ is an upper test function for $u$ at $\left(t_{0}, z_{0}\right) \in \Omega_{T}$ then $d d^{c} q_{t_{0}}\left(z_{0}\right) \geq 0$; b) If $u$ is a subsolution to (5) then $u(t, z)$ is plurisubharmonic in $z$ for every $t \in(0, T)$ (see [4, Corollary 3.7]).

Denote by $\mathcal{S}_{2 n}$ the space of all $2 n \times 2 n$ symmetric matrices. For each function $u: \Omega_{T} \longrightarrow$ $\mathbb{R}$ and for every $\left(t_{0}, z_{0}\right) \in \Omega_{T}$, we define by $\mathcal{P}^{2,+} u\left(t_{0}, z_{0}\right)$ the set of $(\tau, p, Q) \in \mathbb{R} \times \mathbb{R}^{2 n} \times \mathcal{S}_{2 n}$ satisfying

(6) $u(t, z) \leq u\left(t_{0}, z_{0}\right)+\tau\left(t-t_{0}\right)+o\left(\left|t-t_{0}\right|\right)+\left\langle p, z-z_{0}\right\rangle+\frac{1}{2}\left\langle Q\left(z-z_{0}\right), z-z_{0}\right\rangle+o\left(\left|z-z_{0}\right|^{2}\right)$,

and denote by $\overline{\mathcal{P}}^{2,+} u\left(t_{0}, z_{0}\right)$ the set of $(\tau, p, Q) \in \mathbb{R} \times \mathbb{R}^{2 n} \times \mathcal{S}_{2 n}$ satisfying: $\exists\left(t_{m}, z_{m}\right) \rightarrow$ $\left(t_{0}, z_{0}\right)$ and $\left(\tau_{m}, p_{m}, Q_{m}\right) \in \mathcal{P}^{2,+} u\left(t_{0}, z_{0}\right)$ such that $\left(\tau_{m}, p_{m}, Q_{m}\right) \rightarrow(\tau, p, Q)$ and $u\left(t_{m}, z_{m}\right) \rightarrow$ $\left.u\left(t_{0}, z_{0}\right)\right\}$.

We define in the same way the sets $\mathcal{P}^{2,-} u\left(t_{0}, z_{0}\right)$ and $\overline{\mathcal{P}}^{2,-} u\left(t_{0}, z_{0}\right)$ by

$$
\mathcal{P}^{2,-} u\left(t_{0}, z_{0}\right)=-\mathcal{P}^{2,+}(-u)\left(t_{0}, z_{0}\right),
$$

and

$$
\overline{\mathcal{P}}^{2,-} u\left(t_{0}, z_{0}\right)=-\overline{\mathcal{P}}^{2,+}(-u)\left(t_{0}, z_{0}\right) .
$$

Since $F$ and $f$ are continuous, by [3, Proposition 2.6], we have:

\section{Proposition 2.4.}

1. An upper semi-continuous function $u: \Omega_{T} \longrightarrow \mathbb{R}$ is a subsolution to the parabolic equation

$$
\left(d d^{c} u\right)^{n}=e^{\partial_{t} u+F(t, z, u)} \mu(t, z),
$$

if and only if for all $\left(t_{0}, z_{0}\right) \in \Omega_{T}$ and $(\tau, p, Q) \in \overline{\mathcal{P}}^{2,+} u\left(t_{0}, z_{0}\right)$, we have $d d^{c} Q \geq 0$ and

$$
\left(d d^{c} Q\right)^{n} \geq e^{\tau+F\left(t_{0}, z_{0}, u\left(t_{0}, z_{0}\right)\right)} \mu\left(t_{0}, z_{0}\right) .
$$

Here $d d^{c} Q:=\left(d d^{c}\langle Q z, z\rangle\right), z \in \mathbb{C}^{n}=\mathbb{R}^{2 n}$.

2. A lower semi-continuous function $v: \Omega_{T} \longrightarrow \mathbb{R}$ is a supersolution to the parabolic equation (7) if and only if for all $\left(t_{0}, z_{0}\right) \in \Omega_{T}$ and $(\tau, p, Q) \in \overline{\mathcal{P}}^{2,-} u\left(t_{0}, z_{0}\right)$ such that $d d^{c} Q \geq 0$, we have

$$
\left(d d^{c} Q\right)^{n} \leq e^{\tau+F\left(t_{0}, z_{0}, v\left(t_{0}, z_{0}\right)\right)} \mu\left(t_{0}, z_{0}\right) .
$$

The following theorem is the parabolic Jensen-Ishii's maximum principle which plays an important role in the theory of viscosity solution:

Theorem 2.5. [1, Theorem 8.3] Let $u \in U S C\left(\Omega_{T}\right)$ and $v \in L S C\left(\Omega_{T}\right)$. Let $\phi$ be a function defined in $(0, T) \times \Omega^{2}$ such that $(t, \xi, \eta) \longmapsto \phi(t, \xi, \eta)$ is continuously differentiable in $t$ and twice continuously differentiable in $(\xi, \eta)$.

Assume that the function $(t, \xi, \eta) \longmapsto u(t, \xi)-v(t, \eta)-\phi(t, \xi, \eta)$ has a local maximum at some point $(\hat{t}, \hat{\xi}, \hat{\eta}) \in(0, T) \times \Omega^{2}$. 
Assume furthermore that both $w=u$ and $w=-v$ satisfy:

$$
\left\{\begin{aligned}
& \forall(s, z) \in \Omega \quad \exists r>0 \text { such that } \forall M>0 \exists C \text { satisfying } \\
&|(t, \xi)-(s, z)| \leq r \\
&(\tau, p, Q) \in \mathcal{P}^{2,+} w(t, \xi) \\
&|w(t, \xi)|+|p|+|Q| \leq M
\end{aligned}\right\} \Longrightarrow \tau \leq C .
$$

Then for any $\kappa>0$, there exists $\left(\tau_{1}, p_{1}, Q^{+}\right) \in \overline{\mathcal{P}}^{2,+} u(\hat{t}, \hat{\xi}),\left(\tau_{2}, p_{2}, Q^{-}\right) \in \overline{\mathcal{P}}^{2,-} v(\hat{t}, \hat{\eta})$ such that

and

$$
\tau_{1}=\tau_{2}+D_{t} \phi(\hat{t}, \hat{\xi}, \hat{\eta}), p_{1}=D_{\xi} \phi(\hat{t}, \hat{\xi}, \hat{\eta}), p_{2}=-D_{\eta} \phi(\hat{t}, \hat{\xi}, \hat{\eta})
$$

$$
-\left(\frac{1}{\kappa}+\|A\|\right) I \leq\left(\begin{array}{cc}
Q^{+} & 0 \\
0 & -Q^{-}
\end{array}\right) \leq A+\kappa A^{2}
$$

where $A:=D_{\xi, \eta}^{2} \phi(\hat{t}, \hat{\xi}, \hat{\eta}) \in \mathcal{S}_{4 n}$.

The following lemma is deduced by combining Proposition 2.4 and Theorem 2.5:

Lemma 2.6. Let $\left(u_{\tau}\right)$ be a locally uniformly bounded family of real valued functions defined in $\Omega_{T}$.

1. Assume that for every $\tau, u_{\tau}$ is a viscosity subsolution to the equation

$$
\left(d d^{c} w\right)^{n}=e^{\partial_{t} w+F(t, z, w)} \mu(t, z),
$$

in $\Omega_{T}$. Then $\bar{u}=\left(\sup _{\tau} u_{\tau}\right)^{*}$ is a subsolution to (10) in $\Omega_{T}$. Here $\left(\sup _{\tau} u_{\tau}\right)^{*}$ is the upper semicontinuous regularization of $\sup _{\tau} u_{\tau}$.

2. Assume that for every $\tau, u_{\tau}$ is a viscosity supersolution to (10). Then $\underline{u}=\left(\inf _{\tau} u_{\tau}\right)_{*}$ is a supersolution to (10) in $\Omega_{T}$. Here $\left(\inf _{\tau} u_{\tau}\right)_{*}$ is the lower semicontinuous regularization of $\inf _{\tau} u_{\tau}$.

3. If $\tau \in \mathbb{N}$ then 1. and 2. hold for $\bar{u}=\left(\limsup _{\tau \rightarrow \infty} u_{\tau}\right)^{*}$ and $\underline{u}=\left(\liminf _{\tau \rightarrow \infty} u_{\tau}\right)_{*}$.

In the theory of viscosity solution, the comparison principle and Perron method are two key tools for studying the existence and uniqueness of solution. The following comparison principle has been established in [4]:

Theorem 2.7. [4, pages 949-953] Let u (resp. v) be a bounded subsolution (resp. supersolution) to the parabolic complex Monge-Ampère equation (5) in $\Omega_{T}$. Assume that one of the following conditions is satisfied

a) $\mu(t, z)>0$ for every $(t, z) \in(0, T) \times \Omega$.

b) $\mu$ is independent of $t$.

c) Either $u$ or $v$ is locally Lipschitz in $t$ uniformly in $z$.

Then

$$
\sup _{\Omega_{T}}(u-v) \leq \sup _{\partial_{P}\left(\Omega_{T}\right)}(u-v)_{+},
$$

where $\partial_{P}\left(\Omega_{T}\right)=(\{0\} \times \bar{\Omega}) \cup((0, T) \times \partial \Omega)$ is the parabolic boundary of $\Omega_{T}$ and $u$ (resp. $v)$ has been extended as an upper (resp. a lower) semicontinuous function to $\overline{\Omega_{T}}$.

In order to recall the Perron method, we need the concepts of $\epsilon$-sub/super-barrier.

Definition 2.8. a) A function $u \in U S C([0, T) \times \bar{\Omega})$ is called $\epsilon$-subbarrier for (1) if $u$ is subsolution to (5) in the viscosity sense such that $u_{0}-\epsilon \leq u_{*} \leq u \leq u_{0}$ in $\{0\} \times \bar{\Omega}$ and $\varphi-\epsilon \leq u_{*} \leq u \leq \varphi$ in $[0, T) \times \partial \Omega$.

b) A function $u \in \operatorname{LSC}([0, T) \times \bar{\Omega})$ is called $\epsilon$-superbarrier for (1) if $u$ is supersolution to 
(5) in the viscosity sense such that $u_{0}+\epsilon \geq u^{*} \geq u \geq u_{0}$ in $\{0\} \times \bar{\Omega}$ and $\varphi+\epsilon \geq u^{*} \geq u \geq \varphi$ in $[0, T) \times \partial \Omega$.

Proposition 2.9. [2, Proposition 4.1] For all $\epsilon>0$, there exists a continuous $\epsilon$-subbarrier for (1) which is Lipschitz in $t$.

Definition 2.10. We say that $\left(u_{0}, \mu(0,).\right)$ is admissible if for all $\epsilon>0$, there exist $u_{\epsilon} \in C(\bar{\Omega})$ and $C_{\epsilon}>0$ such that $u_{0} \leq u_{\epsilon} \leq u_{0}+\epsilon$ and $\left(d d^{c} u_{\epsilon}\right)^{n} \leq e^{C_{\epsilon}} \mu(0, z)$ in the viscosity sense.

Proposition 2.11. [2, Theorem 1.3] If $\left(u_{0}, \mu(0,).\right)$ is admissible then the function $u_{\epsilon}$ in the definition 2.10 can be taken to be psh in $\Omega$.

Proposition 2.12. [2, Proposition 4.3] If $\left(u_{0}(z), \mu(0, z)\right)$ is admissible then for all $\epsilon>0$, there exists a continuous $\epsilon$-superbarrier for (1) which is Lipschitz in $t$.

Lemma 2.13. (Perron method)[2, Lemma 2.12] Assume that for every $\epsilon>0$, the problem (1) admits a continuous $\epsilon$-superbarrier which is Lipschitz in $t$ and a continuous $\epsilon$ subbarrier. Denote by $S$ the family of all continuous subsolutions to (1). Then $\phi_{S}=$ $\sup \{v: v \in S\}$ is a discontinuous viscosity solution to $(1)$, i.e., $\left(\phi_{S}\right)^{*}$ is a subsolution and $\left(\phi_{S}\right)_{*}$ is a supersolution.

\section{Some useful Lemmas}

Throughout this section, we always suppose that $\Phi$ and $f$ satisfy the conditions as in Theorem 1.1. Given a bounded function $u: \Omega_{T} \rightarrow \mathbb{R}$ and a constant $A>2 \operatorname{osc}_{\Omega_{T}}(u)$. For every relatively compact open subet $U$ of $\Omega$ and for each constant $0<\delta \ll 1$ satisfying $\Phi([-\delta, \delta] \times U) \subset \Omega)$, we define

and

$$
u^{k}(t, z)=\sup \left\{u(t+s, \Phi(s, z))-k|s|:|s| \leq \frac{A}{k}\right\}
$$

$$
u_{k}(t, z)=\inf \left\{u(t+s, \Phi(s, z))+k|s|:|s| \leq \frac{A}{k}\right\}
$$

for every $k>\max \left\{\frac{A}{\delta}, \frac{2 A}{T}\right\}$ and $(t, z) \in(A / k, T-A / k) \times U$.

We have the following modified version of [4, Lemma 3.5]:

Lemma 3.1. Assume that $u$ is a bounded upper semicontinuous function in $\Omega_{T}$. Then

(i) $u^{k}$ is upper semicontinuous in $(A / k, T-A / k) \times U$;

(ii) for all $(t, z) \in(A / k, T-A / k) \times U$,

$$
u(t, z) \leq u^{k}(t, z) \leq \sup _{|s| \leq A / k} u(t+s, \Phi(s, z)) ;
$$

(iii) if $(t, z)$ and $(t+s, \Phi(s, z))$ belong in $(A / k, T-A / k) \times U$ then

$$
\left|u^{k}(t, z)-u^{k}(t+s, \Phi(s, z))\right| \leq k|s|
$$

(iv) if $\left(d d^{c} u\right)^{n} \geq e^{\partial_{t} u+F(t, z, u)} \mu(t, z)$ in the viscosity sense in $\Omega_{T}$ then, for every $0<\epsilon<$ 1 , there exists $k_{\epsilon}>0$ such that, for every $k>k_{\epsilon}$,

$$
\left(d d^{c} u^{k}\right)^{n} \geq(1-\epsilon) e^{\partial_{t} u^{k}+F_{k}\left(t, z, u^{k}\right)} f_{k}(t, z) d V,
$$

in the viscosity sense in $(\delta, T-\delta) \times U$, where $F_{k}(t, z, r)=\inf _{|s| \leq A / k} F(t+s, \Phi(s, z), r)$ and $f_{k}(t, z)=\inf _{|s| \leq A / k} f(t+s, \Phi(s, z))$.

Proof. (i) Let $\left(t_{0}, z_{0}\right) \in(A / k, T-A / k) \times U$. We will show that 


$$
u^{k}\left(t_{0}, z_{0}\right) \geq \limsup _{(t, z) \rightarrow\left(t_{0}, z_{0}\right)} u^{k}(t, z) .
$$

Assume that $\left(t_{m}, z_{m}\right) \in(A / k, T-A / k) \times U$ satisfies $\left(t_{m}, z_{m}\right) \rightarrow\left(t_{0}, z_{0}\right)$ as $m \rightarrow \infty$ and

$$
\limsup _{(t, z) \rightarrow\left(t_{0}, z_{0}\right)} u^{k}(t, z)=\lim _{m \rightarrow \infty} u^{k}\left(t_{m}, z_{m}\right) .
$$

Since $u$ is usc and $\Phi$ is continuous, by the definition of $u^{k}$, we have

$$
u^{k}\left(t_{m}, z_{m}\right)=u\left(s_{m}+t_{m}, \Phi\left(s_{m}, z_{m}\right)\right)-k\left|s_{m}\right|,
$$

for some $\left|s_{m}\right| \leq A / k$. Let $\left\{s_{m_{l}}\right\}$ be a subsequence of $\left\{s_{m}\right\}$ which converges to a point $s_{0} \in[-A / k, A / k]$. Then

$$
\begin{aligned}
\limsup _{(t, z) \rightarrow\left(t_{0}, z_{0}\right)} u^{k}(t, z) & =\lim _{m_{l} \rightarrow \infty} u^{k}\left(t_{m_{l}}, z_{m_{l}}\right) \\
& =\lim _{m_{l} \rightarrow \infty}\left(u\left(s_{m_{l}}+t_{m_{l}}, \Phi\left(s_{m_{l}}, z_{m_{l}}\right)\right)-k\left|s_{m_{l}}\right|\right) \\
& =\lim _{m_{l} \rightarrow \infty} u\left(s_{m_{l}}+t_{m_{l}}, \Phi\left(s_{m_{l}}, z_{m_{l}}\right)\right)-k\left|s_{0}\right| \\
& \leq u\left(s_{0}+t_{0}, \Phi\left(s_{0}, z_{0}\right)\right)-k\left|s_{0}\right| \\
& \leq u^{k}\left(t_{0}, z_{0}\right) .
\end{aligned}
$$

Hence, $u^{k}$ is usc in $(A / k, T-A / k) \times U$.

(ii) Obvious.

(iii) Let $\left|s_{0}\right| \leq A / k$ such that $u^{k}(t, z)=u\left(t+s_{0}, \Phi\left(s_{0}, z\right)\right)-k\left|s_{0}\right|$. If $\left|s-s_{0}\right|>A / k$ then

$$
\begin{aligned}
u^{k}(t, z)=u\left(t+s_{0}, \Phi\left(s_{0}, z\right)\right)-k\left|s_{0}\right| & \leq u(t+s, \Phi(s, z))+2 o s c_{\Omega_{T}} u-k\left|s_{0}\right| \\
& \leq u^{k}(t+s, \Phi(s, z))+A-k\left|s_{0}\right| \\
& \leq u^{k}(t+s, \Phi(s, z))+k\left|s-s_{0}\right|-k\left|s_{0}\right| \\
& \leq u^{k}(t+s, \Phi(s, z))+k|s| .
\end{aligned}
$$

If $\left|s-s_{0}\right| \leq A / k$ then

$$
\begin{aligned}
u^{k}(t, z)=u\left(t+s_{0}, \Phi\left(s_{0}, z\right)\right)-k\left|s_{0}\right| & \leq u^{k}(t+s, \Phi(s, z))+k\left|s-s_{0}\right|-k\left|s_{0}\right| \\
& \leq u^{k}(t+s, \Phi(s, z))+k|s| .
\end{aligned}
$$

Hence

$$
u^{k}(t, z)-u^{k}(t+s, \Phi(s, z)) \leq k|s|
$$

By the same argument, we also have

$$
u^{k}(t+s, \Phi(s, z))-u^{k}(t, z) \leq k|s|
$$

Therefore

$$
\left|u^{k}(t, z)-u^{k}(t+s, \Phi(s, z))\right| \leq k|s|
$$

(iv) Let $r_{0}>0$ such that $V:=U+r_{0} \mathbb{B}^{2 n} \Subset \Omega$. Since $\Phi(t, z)$ is holomorphic in $z$ and converges locally uniformly to $I d$ as $t \rightarrow 0$, we have $\frac{\partial \Phi_{\alpha}}{\partial z_{\beta}}(t, z)$ converges uniformly in $V$ to $\delta_{\alpha \beta}$ as $t \rightarrow 0$ for every $1 \leq \alpha, \beta \leq n$. Hence, for every $0<\epsilon<1$, there exists $0<r_{1}<\delta$ such that $\Phi\left(\left[-r_{1}, r_{1}\right] \times V\right) \Subset \Omega$ and

$$
\left|\operatorname{det}\left(\frac{\partial \Phi_{j}}{\partial z_{k}}(t, z)\right)\right|^{2}>1-\epsilon
$$

for every $(t, z) \in\left[-r_{1}, r_{1}\right] \times V$. Denote $k_{\epsilon}=\max \left\{A / r_{1}, 2 A / T\right\}$. We will show that (11) holds in the viscosity sense in $(\delta, T-\delta) \times U$ for every $k>k_{\epsilon}$. 
Let $\left(t_{0}, z_{0}\right) \in(\delta, T-\delta) \times U, s_{0} \in(-A / k, A / k)$ and let $q$ be an upper test function of $u_{s_{0}}(t, z):=u\left(t+s_{0}, \Phi\left(s_{0}, z\right)\right)-k\left|s_{0}\right|$ at $\left(t_{0}, z_{0}\right)$. Then $\hat{q}(t, z):=q\left(t-s_{0}, \Phi^{-1}\left(s_{0}, z\right)\right)+k\left|s_{0}\right|$ is an upper test function of $u$ at $(\hat{t}, \hat{z})=\left(t_{0}+s_{0}, \Phi\left(s_{0}, z_{0}\right)\right)$. Since $\left(d d^{c} u\right)^{n} \geq e^{\partial_{t} u+F(t, z, u)} \mu(t, z)$ in the viscosity sense, we have

$$
\left.\left(d d^{c} \hat{q}(\hat{t}, \xi)\right)^{n}\right|_{\xi=\hat{z}} \geq e^{\partial_{t} \hat{q}(\hat{t}, \hat{z})+F(\hat{t}, \hat{z}, \hat{q}(\hat{t}, \hat{z}))} \mu(\hat{t}, \hat{z}) .
$$

Note that $\partial_{t} \hat{q}(\hat{t}, \hat{z})=\partial_{t} q\left(t_{0}, z_{0}\right)$ and

$$
\left.\left(d d^{c} q\left(t_{0}, \xi\right)\right)^{n}\right|_{\xi=z_{0}}=\left.\left|\operatorname{det}\left(\frac{\partial \Phi_{j}}{\partial z_{k}}(t, z)\right)\right|^{2}\left(d d^{c} \hat{q}(\hat{t}, \xi)\right)^{n}\right|_{\xi=\hat{z}} .
$$

Therefore, by (12) and (13), we have

$$
\begin{aligned}
\left.\left(d d^{c} q\left(t_{0}, \xi\right)\right)^{n}\right|_{\xi=z_{0}} & \geq(1-\epsilon) e^{\partial_{t} q\left(t_{0}, z_{0}\right)+F(\hat{t}, \hat{z}, \hat{q}(\hat{t}, \hat{z}))} \mu(\hat{t}, \hat{z}) \\
& \geq(1-\epsilon) e^{\partial_{t} q\left(t_{0}, z_{0}\right)+F\left(\hat{t}, \hat{z}, q\left(t_{0}, z_{0}\right)\right)} \mu(\hat{t}, \hat{z}) \\
& \geq(1-\epsilon) e^{\partial_{t} q\left(t_{0}, z_{0}\right)+F_{k}\left(t_{0}, z_{0}, q\left(t_{0}, z_{0}\right)\right)} f_{k}\left(t_{0}, z_{0}\right) d V .
\end{aligned}
$$

Since $\left(t_{0}, z_{0}\right)$ and $q$ are arbitrary, we get $u_{s_{0}}$ is a subsolution to the equation

$$
\left(d d^{c} w\right)^{n}=(1-\epsilon) e^{\partial_{t} w+F_{k}(t, z, w)} f_{k}(t, z) d V,
$$

in $(\delta, T-\delta) \times U$. Then, it follows from Lemma 2.6 that the function

$$
u^{k}=\sup _{\left|s_{0}\right| \leq A / k} u_{s_{0}}=\left(\sup _{\left|s_{0}\right| \leq A / k} u_{s_{0}}\right)^{*}
$$

is a subsolution to $(14)$ in $(\delta, T-\delta) \times U$.

The proof is completed.

By the same argument, we have

Lemma 3.2. Assume that $u$ is a bounded lower semicontinuous function in $\Omega_{T}$. Then

(i) $u_{k}$ is lower semicontinuous in $(A / k, T-A / k) \times U$;

(ii) for all $(t, z) \in(A / k, T-A / k) \times U$,

$$
u(t, z) \geq u_{k}(t, z) \geq \inf _{|s| \leq A / k} u(t+s, \Phi(s, z))
$$

(iii) if $(t, z)$ and $(t+s, \Phi(s, z))$ belong in $(A / k, T-A / k) \times U$ then

$$
\left|u_{k}(t, z)-u_{k}(t+s, \Phi(s, z))\right| \leq k|s|
$$

(iv) if $\left(d d^{c} u\right)^{n} \leq e^{\partial_{t} u+F(t, z, u)} \mu(t, z)$ in the viscosity sense in $\Omega_{T}$ then, for every $0<\epsilon<$ 1 , there exists $k_{\epsilon}>0$ such that, for every $k>k_{\epsilon}$,

$$
\left(d d^{c} u_{k}\right)^{n} \leq(1+\epsilon) e^{\partial_{t} u_{k}+F^{k}\left(t, z, u_{k}\right)} f^{k}(t, z) d V
$$

in the viscosity sense in $(\delta, T-\delta) \times U$, where $F^{k}(t, z, r)=\sup _{|s| \leq A / k} F(t+$ $s, \Phi(s, z), r)$ and $f^{k}(t, z)=\sup _{|s| \leq A / k} f(t+s, \Phi(s, z))$.

In [4], by applying the maximum principle, Eyssidieux-Guedj-Zeriahi have proved the comparison principle for the case where either the given subsolution or the given supersolution is Lipschitz in $t$. Using the same method as in [4], we obtain the following lemma:

Lemma 3.3. Suppose that $f$ satisfies the conditions in Theorem 1.1. Let $u \in U S C \cap$ $L^{\infty}([0, T) \times \bar{\Omega})$ and $v \in L S C \cap L^{\infty}([0, T) \times \bar{\Omega})$ be, respectively, a subsolution and a supersolution to the equation

$$
\left(d d^{c} w\right)^{n}=e^{\partial_{t} w+F(t, z, w)} \mu(t, z),
$$


in $\Omega_{T}$. Assume that, for $w=u, v$, the following condition holds: for every $U \Subset \Omega$ and $0<\delta<T / 2$, there exists $k(U, \delta)>0$ such that if $(t, z) \in(\delta, T-\delta) \times U$ then

$$
|w(t, z)-w(t+s, \Phi(s, z))| \leq k(U, \delta)|s|,
$$

for $0<|s| \ll 1$. Then

$$
\sup _{\Omega_{T}}(u-v) \leq \sup _{\partial_{P} \Omega_{T}}(u-v)_{+} \cdot
$$

Proof. Let $\delta>0$ be an arbitrary positive constant and denote

$$
h(t, z)=u(t, z)-v(t, z)-\frac{\delta}{T-t}+\delta\left(|z|^{2}-C\right),
$$

where $C=\sup _{z \in \Omega}|z|^{2}$. We will show that

$$
\max _{\overline{\Omega_{T}}} h \leq \max _{\partial_{P} \Omega_{T}} h_{+} .
$$

Assume that (17) is false. Then, there exists $\left(t_{0}, z_{0}\right) \in \Omega_{T}$ such that

$$
M:=h\left(t_{0}, z_{0}\right)=\max _{[0, T) \times \bar{\Omega}} h>\max _{\partial_{P} \Omega_{T}} h_{+} .
$$

It follows from [4, Lemma 4.1] that

$$
f\left(t_{0}, z_{0}\right)=0 .
$$

For every $N>0$, we denote

$h_{N}(t, \xi, \eta)=u(t, \xi)+\delta\left(|\xi|^{2}-C\right)-v(t, \eta)-\frac{\delta}{T-t}-\frac{N\left(\left|\xi-z_{0}\right|^{2}+\left|\eta-z_{0}\right|^{2}\right)}{2}-\frac{N^{2}\left|t-t_{0}\right|^{2}}{2}$,

and let $\left(t_{N}, \xi_{N}, \eta_{N}\right) \in[0, T) \times \bar{\Omega}^{2}$ such that

$$
h_{N}\left(t_{N}, \xi_{N}, \eta_{N}\right)=\max _{[0, T) \times \bar{\Omega}^{2}} h_{N}=: M_{N} .
$$

By [1, Proposition 3.7], we have

$$
\lim _{N \rightarrow \infty}\left(N^{2}\left|t_{N}-t_{0}\right|^{2}+N\left|\xi_{N}-z_{0}\right|^{2}+N\left|\eta_{N}-z_{0}\right|^{2}\right)=0 .
$$

In particular, there exists $N_{0}>0$ such that $\left(t_{N}, \xi_{N}, \eta_{N}\right) \in(0, T) \times \Omega^{2}$ for all $N \geq N_{0}$.

By Lemma 3.4 below, the functions $w_{1}=u+\delta\left(|z|^{2}-C\right)$ and $w_{2}=-v$ satisfy the condition (2.5) in Theorem 2.5. Then, it follows from Theorem 2.5 that, for every $N>N_{0}$, there exist $\left(\tau_{N 1}, p_{N 1}, Q_{N}^{+}\right) \in \overline{\mathcal{P}}^{2,+} w_{1}\left(t_{N}, \xi_{N}\right)$ and $\left(\tau_{N 2}, p_{N 2}, Q_{N}^{-}\right) \in \overline{\mathcal{P}}^{2,-} v\left(t_{N}, \eta_{N}\right)$ such that

$$
p_{N 1}=N\left(\xi_{N}-z_{0}\right), \quad p_{N 2}=-N\left(\eta_{N}-z_{0}\right),
$$

and $Q_{N}^{-} \geq Q_{N}^{+}$(i.e., $\left\langle Q_{N}^{-} \zeta, \zeta\right\rangle \geq\left\langle Q_{N}^{+} \zeta, \zeta\right\rangle$ for every $\zeta \in \mathbb{R}^{2 n}$ ). In particular, we have

$$
d d^{c} Q_{N}^{-} \geq d d^{c} Q_{N}^{+} \geq \delta \omega>0,
$$

where $\omega=d d^{c}|z|^{2}$. The second inequality holds due to Proposition 2.4. Moreover, it follows from Proposition 2.4 that

$$
e^{\tau_{N 2}+F\left(t_{N}, \eta_{N}, v\left(t_{N}, \eta_{N}\right)\right)} \mu\left(t_{N}, \eta_{N}\right) \geq\left(d d^{c} Q_{N}^{-}\right)^{n} .
$$

Combining (21) and (22), we get

$$
e^{\tau_{N 2}+F\left(t_{N}, \eta_{N}, v\left(t_{N}, \eta_{N}\right)\right)} \mu\left(t_{N}, \eta_{N}\right) \geq \delta^{n} \omega^{n} .
$$


Since $F(t, z, v(t, z))$ is bounded, it follows that there exists $m>0$ such that

$$
e^{\tau_{N 2}} f\left(t_{N}, \eta_{N}\right) \geq m
$$

for every $N \gg 1$.

Since $f\left(t_{0}, z_{0}\right)=0$, the condition (4) follows that $f\left(t_{N}, z_{N}\right)=0$, where $z_{N}=\Phi\left(t_{N}-\right.$ $\left.t_{0}, z_{0}\right)$. Then, by the condition (3), there exist $a, b>0$ such that

$$
f\left(t_{N}, \eta_{N}\right) \leq \exp \left(b-a \frac{\left|\left\langle z_{N}-\eta_{N}, \partial_{t} \Phi\left(0, \eta_{N}\right)\right\rangle\right|}{\left|z_{N}-\eta_{N}\right|^{2}}\right)
$$

for every $N \gg 1$.

By the assumption on $\Phi$, there exists $C_{1}>0$ such that

$$
\left|z_{N}-z_{0}\right| \leq C_{1}\left|t_{N}-t_{0}\right|=o\left(\frac{1}{N}\right)
$$

where the last estimate holds due to (19). Combining (25) and (19), we get that

$$
\lim _{N \rightarrow \infty} N\left|z_{N}-\eta_{N}\right|^{2}=0
$$

By (20) and by Lemma 3.4 below, we have

$$
\tau_{N 2}=O(1)-N\left\langle\eta_{N}-z_{0}, \partial_{t} \Phi\left(0, \eta_{N}\right)\right\rangle
$$

Combining (27) and (25), we get

$$
\tau_{N 2}=O(1)-N\left\langle\eta_{N}-z_{N}, \partial_{t} \Phi\left(0, \eta_{N}\right)\right\rangle .
$$

Combining (24), (28) and (26), we get

$$
e^{2 \tau_{N 2}} f\left(t_{N}, \eta_{N}\right)=O(1) .
$$

Since $\lim _{N \rightarrow \infty} f\left(t_{N}, \eta_{N}\right)=f\left(t_{0}, z_{0}\right)=0$, it follows that

$$
\lim _{N \rightarrow \infty} e^{2 \tau_{N 2}} f^{2}\left(t_{N}, \eta_{N}\right)=0,
$$

and it contradicts with (23). Then

$$
\max _{\overline{\Omega_{T}}} h \leq \max _{\partial_{P} \Omega_{T}} h_{+} .
$$

Letting $\delta \searrow 0$, we obtain

$$
\sup _{\Omega_{T}}(u-v) \leq \sup _{\partial_{P} \Omega_{T}}(u-v)_{+} \cdot
$$

The proof is completed.

Lemma 3.4. Let $w$ be a bounded usc function in $\Omega_{T}$ satisfying the following condition: for every $U \Subset \Omega$ and $0<\delta<T / 2$, there exists $k(U, \delta)>0$ such that if $(t, z) \in(\delta, T-\delta) \times U$ then

$$
|w(t, z)-w(t+s, \Phi(s, z))| \leq k(U, \delta)|s|,
$$

for $0<|s| \ll 1$. Then $w$ satisfies the condition (2.5) in Theorem 2.5. Moreover,

$$
\left|\tau-\left\langle p, \partial_{t} \Phi\left(0, z_{0}\right)\right\rangle\right| \leq k(U, \delta)
$$

for every $\left(t_{0}, z_{0}\right) \in(\delta, T-\delta) \times U$ and $(\tau, p, Q) \in \mathcal{P}^{2,+} w\left(t_{0}, z_{0}\right)$.

Proof. Assume that $q$ is an upper test function for $w$ at $\left(t_{0}, z_{0}\right) \in(\delta, T-\delta) \times U$. By (29), for $0<|s| \ll 1$, we have

$$
q\left(s+t_{0}, \Phi\left(s, z_{0}\right)\right)-q\left(t_{0}, z_{0}\right) \geq-k(U, \delta)|s| .
$$


Then, for $0<|s| \ll 1$,

$$
\left|\frac{q\left(s+t_{0}, \Phi\left(s, z_{0}\right)\right)-q\left(t_{0}, z_{0}\right)}{s}\right| \leq k(U, \delta) .
$$

Letting $s \rightarrow 0$, we get

$$
\left|\frac{\partial q}{\partial t}\left(t_{0}, z_{0}\right)-\left\langle\nabla q\left(t_{0}, z_{0}\right), \partial_{t} \Phi\left(0, z_{0}\right)\right\rangle\right| \leq k(U, \delta) .
$$

Note that $(\tau, p, Q) \in \mathcal{P}^{2,+} w\left(t_{0}, z_{0}\right)$ iff there exists an upper test function $q$ for $w$ at $\left(t_{0}, z_{0}\right)$ such that $(\tau, p, Q)=\left(\partial_{t} q\left(t_{0}, z_{0}\right), D q\left(t_{0}, z_{0}\right), D^{2} q\left(t_{0}, z_{0}\right)\right)$. Then, (30) is satisfied, and it is easy to see that $w$ satisfies the condition (2.5) in Theorem 2.5.

\section{Proof of Theorem 1.1 And Corollary 1.2}

For the reader's convenience, we recall Theorem 1.1:

Theorem 4.1. Let $\Phi:(-1,1) \times \Omega \rightarrow \mathbb{C}^{n}$ be a continuous mapping satisfying the following conditions:

- the mapping $z \mapsto \Phi(s, z)$ is holomorphic in $\Omega$ for every $s \in(-1,1)$;

- $\Phi(0, z)=z$ for every $z \in \Omega$;

- $\partial_{t} \Phi$ is well defined and continuous on $(-1,1) \times \Omega$.

Suppose that $f$ satisfies the following conditions:

- for every $0<R<S<T$ and $K \Subset \Omega$, there exist $a, b>0$ such that if $\left(t_{0}, z_{0}\right) \in$ $(R, S) \times K$ and $f\left(t_{0}, z_{0}\right)=0$ then

$$
f\left(t_{0}, z\right) \leq \exp \left(b-a \frac{\left|\left\langle z-z_{0}, \partial_{t} \Phi\left(0, z_{0}\right)\right\rangle\right|}{\left|z-z_{0}\right|^{2}}\right)
$$

for every $z \in \Omega \backslash\left\{z_{0}\right\}$;

- for every $0<R<S<T, K \Subset \Omega$ and $\epsilon>0$, there exists $0<\delta<\delta_{K}$ such that

$$
(1+\epsilon) f(t, z) \geq f(t+s, \Phi(s, z)),
$$

for every $z \in K, R<t<S$ and $|s|<\delta$.

Assume that $u$ and $v$, respectively, is a bounded viscosity subsolution and a bounded viscosity supersolution to the Cauchy-Dirichlet problem (1). Then, for every $0<R<S<T$, $K \Subset \Omega$ and $\epsilon>0$, there exists $0<\delta \ll 1$ such that

$$
u\left(t+s_{1}, \Phi\left(s_{1}, z\right)\right)<v\left(t+s_{2}, \Phi\left(s_{2}, z\right)\right)+\epsilon,
$$

for all $z \in K, R<t<S$ and $\max \left\{\left|s_{1}\right|,\left|s_{2}\right|\right\}<\delta$.

Proof. Let $0<R<S<T$ and denote $S_{1}=(S+T) / 2$. First, we show that for every $\epsilon>0$ there exists $\delta_{1}=\delta_{1}(\epsilon) \in(0, \min \{1, R\})$ such that $K \Subset \Omega_{\delta_{1}}$ and

$$
u(t, \eta)<v(s, \xi)+\epsilon,
$$

for every $(t, \eta),(s, \xi) \in \Omega_{S_{1}} \backslash\left(\left[\delta_{1}, S_{1}\right] \times \Omega_{\delta_{1}}\right)$ with $|t-s|+|\eta-\xi|<\delta_{1}$. Here $\Omega_{\delta_{1}}=\{z \in$ $\left.\Omega: d(z, \partial \Omega)>\delta_{1}\right\}$.

We consider the mapping

$$
\begin{gathered}
G: \overline{\Omega_{S_{1}}} \times \overline{\Omega_{S_{1}}} \longrightarrow \mathbb{R} \\
(t, \eta, s, \xi) \mapsto u(t, \eta)-v(s, \xi) .
\end{gathered}
$$

Since $G$ is upper semicontinuous, the set

$$
U=\left\{(t, \eta, s, \xi) \in{\overline{\Omega_{S_{1}}}}^{2}: G(t, \eta, s, \xi)<\epsilon\right\},
$$


is relatively open in ${\overline{\Omega_{S_{1}}}}^{2}$. Denote

$$
A=\left\{(t, \eta, t, \eta):(t, \eta) \in\left(\left[0, S_{1}\right] \times \partial \Omega\right) \cup(\{0\} \times \bar{\Omega})\right\} .
$$

We have $A$ is compact and $G \leq 0$ on $A$. Hence there exists $0<\delta_{1}<\min \{1, R, \operatorname{dist}(K, \partial \Omega)\}$ such that

$$
{\overline{\Omega_{S_{1}}}}^{2} \cap\left(3 \delta_{1} \mathbb{B}_{4 n+2}+A\right) \subset U,
$$

where $\mathbb{B}_{4 n+2}$ is the unit ball in the Euclidean space $\mathbb{R}^{4 n+2}$. Then, we have

$$
\left\{(t, \eta, t, \eta):(t, \eta) \in \Omega_{S_{1}} \backslash\left(\left[\delta_{1}, S_{1}\right] \times \Omega_{\delta_{1}}\right)\right\} \subset 2 \delta_{1} \mathbb{B}_{4 n+2}+A .
$$

Note that if $|t-s|+|\eta-\xi|<\delta_{1}$ then $(t, \eta, s, \xi)-(t, \eta, t, \eta) \in \delta_{1} \mathbb{B}_{4 n+2}$. Therefore, it follows from (36) that

$$
\left\{(t, \eta, s, \xi) \in\left(\Omega_{S_{1}} \backslash\left(\left[\delta_{1}, S_{1}\right] \times \Omega_{\delta_{1}}\right)\right)^{2}:|t-s|+|\eta-\xi|<\delta_{1}\right\} \subset 3 \delta_{1} \mathbb{B}_{4 n+2}+A .
$$

Combining (35) and (37), we obtain (34). By the condition $0<\delta_{1}<\min \{1, R, \operatorname{dist}(K, \partial \Omega)\}$, we also have $K \Subset \Omega_{\delta_{1}}$.

By the assumption (32), there exists $\delta_{2}=\delta_{2}(\epsilon) \in\left(0, \frac{\min \left\{\delta_{1}, T-S\right\}}{4}\right)$ such that

$$
8\left(C_{\Omega_{\delta_{1} / 2}}+1\right) \delta_{2}<\delta_{1}
$$

and

$$
(1+\epsilon) f(t, z) \geq f(t+s, \Phi(s, z)),
$$

for every $z \in \Omega_{\delta_{1} / 2}, \delta_{1} / 2<t<S_{1}$ and $|s|<2 \delta_{2}$. Here $C_{\Omega_{\delta_{1} / 2}}>0$ is defined by (??). Since $F$ is continuous, we can choose $\delta_{2}$ small enough such that

$$
|F(t, z, r)-F(t+s, \Phi(s, z), r)|<\epsilon,
$$

for every $z \in \Omega_{\delta_{1} / 2}, \delta_{1} / 2<t<S_{1},|s|<2 \delta_{2}$ and $|r|<M$, where $M=\max \left\{\sup _{\Omega_{T}}|u|, \sup _{\Omega_{T}}|v|\right\}$.

For every $k>\frac{2 M}{\delta_{2}}$ and $(t, z) \in\left[\delta_{1} / 2, S_{1}\right] \times \Omega_{\delta_{1} / 2}$, we consider

$$
u^{k}(t, z)=\sup \left\{u(t+s, \Phi(s, z))-k|s|:|s| \leq \frac{2 M}{k}\right\}
$$

and

$$
v_{k}(t, z)=\inf \left\{v(t+s, \Phi(s, z))-k|s|:|s| \leq \frac{2 M}{k}\right\} .
$$

It follows from (34) and (38) that

$$
u^{k}\left(t+\tau_{1}, \Phi\left(\tau_{1}, z\right)\right) \leq v_{k}\left(t+\tau_{2}, \Phi\left(\tau_{2}, z\right)\right)+\epsilon,
$$

for every $(t, z) \in \partial_{P}\left(\left(3 \delta_{1} / 4, S\right) \times \Omega_{3 \delta_{1} / 4}\right)$ and $\max \left\{\left|\tau_{1}\right|,\left|\tau_{2}\right|\right\}<\delta_{2}$. Denote $u_{\tau_{1}}^{k}(t, z)=$ $u^{k}\left(t+\tau_{1}, \Phi\left(\tau_{1}, z\right)\right)$ and $v_{k, \tau_{2}}(t, z)=v_{k}\left(t+\tau_{2}, \Phi\left(\tau_{2}, z\right)\right)$. By Lemma 3.1 and Lemma 3.2, there exists $k_{\epsilon}>\frac{2 M}{\delta_{2}}$ such that

$$
\left(d d^{c} u_{\tau_{1}}^{k}\right)^{n} \geq(1-\epsilon) e^{\partial_{t} u_{\tau_{1}}^{k}+F_{k}\left(t+\tau_{1}, \Phi\left(\tau_{1}, z\right), u_{\tau_{1}}^{k}\right)} f_{k}\left(t+\tau_{1}, \Phi\left(\tau_{1}, z\right)\right) d V
$$

and

$$
\left(d d^{c} v_{k, \tau_{2}}\right)^{n} \leq(1+\epsilon) e^{\partial_{t} v_{k, \tau_{2}}+F^{k}\left(t+\tau_{2}, \Phi\left(\tau_{2}, z\right), v_{k, \tau_{2}}\right)} f^{k}\left(t+\tau_{2}, \Phi\left(\tau_{2}, z\right)\right) d V,
$$

in the viscosity sense in $\left(3 \delta_{1} / 4, S\right) \times \Omega_{3 \delta_{1} / 4}$ for every $k>k_{\epsilon}$ and $\max \left\{\left|\tau_{1}\right|,\left|\tau_{2}\right|\right\}<\delta_{2}$. Here, $F_{k}(t, z, r)=\inf _{|s| \leq 2 M / k} F(t+s, \Phi(s, z), r), f_{k}(t, z)=\inf _{|s| \leq 2 M / k} f(t+s, \Phi(s, z))$, $F^{k}(t, z, r)=\sup _{|s| \leq 2 M / k} F(t+s, \Phi(s, z), r)$ and $f^{k}(t, z)=\sup _{|s| \leq 2 M / k} f(t+s, \Phi(s, z))$. 
Moreover, it follows from (39) and (40) that

$$
(1+\epsilon) f_{k}\left(t+\tau_{1}, \Phi\left(\tau_{1}, z\right)\right) \geq f(t, z) \geq \frac{f^{k}\left(t+\tau_{2}, \Phi\left(\tau_{2}, z\right)\right)}{1+\epsilon},
$$

and

$$
F_{k}\left(t+\tau_{1}, \Phi\left(\tau_{1}, z\right), r\right)+\epsilon \geq F(t, z, r) \geq F^{k}\left(t+\tau_{2}, \Phi\left(\tau_{2}, z\right), r\right)-\epsilon,
$$

for every $(t, z) \in\left(3 \delta_{1} / 4, S\right) \times \Omega_{3 \delta_{1} / 4}, \max \left\{\left|\tau_{1}\right|,\left|\tau_{2}\right|\right\}<\delta_{2},|r| \leq M$ and $k>k_{\epsilon}$.

Combining (42), (43), (44) and (45), we get

$$
\left(d d^{c} u_{\tau_{1}}^{k}\right)^{n} \geq(1-\epsilon)^{2} e^{\partial_{t} u_{\tau_{1}}^{k}+F\left(t, z, u_{\tau_{1}}^{k}\right)-\epsilon} f(t, z) d V
$$

and

$$
\left(d d^{c} v_{k, \tau_{2}}\right)^{n} \leq(1+\epsilon)^{2} e^{\partial_{t} v_{k, \tau_{2}}+F\left(t, z, v_{k, \tau_{2}}\right)+\epsilon} f(t, z) d V,
$$

in the viscosity sense in $\left(3 \delta_{1} / 4, S\right) \times \Omega_{3 \delta_{1} / 4}$ for every $k>k_{\epsilon}$ and $\max \left\{\left|\tau_{1}\right|,\left|\tau_{2}\right|\right\}<\delta_{2}$. Therefore, $w_{1}:=u_{\tau_{1}}^{k}+3 \epsilon t$ and $w_{2}:=v_{k, \tau_{2}}-3 \epsilon t$ is respectively a subsolution and a supersolution to the equation

$$
e^{\partial_{t} w+F(t, z, w)} \mu(t, z)=\left(d d^{c} w\right)^{n},
$$

in $\left(3 \delta_{1} / 4, S\right) \times \Omega_{3 \delta_{1} / 4}$. Note that, by Lemma 3.1 and Lemma 3.2, the functions $w_{1}$ and $w_{2}$ satisfy the condition (16) in Lemma 3.3. Then, by using Lemma 3.3, we have

$$
\sup _{\left(3 \delta_{1} / 4, S\right) \times \Omega_{3 \delta_{1} / 4}}\left(w_{1}-w_{2}\right) \leq \sup _{\partial_{P}\left(\left(3 \delta_{1} / 4, S\right) \times \Omega_{3 \delta_{1} / 4}\right)}\left(w_{1}-w_{2}\right)_{+} \leq \epsilon+6 \epsilon S
$$

where the last inequality holds due to (41). Then

$$
u^{k}\left(t+\tau_{1}, \Phi\left(\tau_{1}, z\right)\right)-v_{k}\left(t+\tau_{2}, \Phi\left(\tau_{2}, z\right)\right) \leq-6 \epsilon t+\epsilon+6 \epsilon S \leq(6 S+1) \epsilon,
$$

in $\left(3 \delta_{1} / 4, S\right) \times \Omega_{3 \delta_{1} / 4}$ for every $k>k_{\epsilon}$ and $|\tau|<\delta_{2}$. Letting $k \rightarrow \infty$, we get

$$
u\left(t+\tau_{1}, \Phi\left(\tau_{1}, z\right)\right)-v\left(t+\tau_{2}, \Phi\left(\tau_{2}, z\right)\right) \leq(6 S+1) \epsilon
$$

in $(R, S) \times K \subset\left(3 \delta_{1} / 4, S\right) \times \Omega_{3 \delta_{1} / 4}$ for every $\max \left\{\left|\tau_{1}\right|,\left|\tau_{2}\right|\right\}<\delta_{2}$. Choosing $\delta=\delta(\epsilon)=$ $\delta_{2}(\epsilon /(6 S+1))$, we obtain $(33)$.

The proof is completed.

Corollary 4.2. Assume that $\Omega$ is a smooth strictly pseudoconvex domain and $\left(u_{0}, \mu(0, z)\right)$ is admissible. Suppose that $\Phi$ and $f$ satisfy the conditions in Theorem 1.1. Then the Cauchy-Dirichlet problem (1) has a unique viscosity solution.

Proof. By Propositions 2.9 and 2.12, for every $\epsilon>0$, the problem (1) admits a continuous $\epsilon$-superbarrier which is Lipschitz in $t$ and a continuous $\epsilon$-subbarrier. Then, it follows from Lemma 2.13 that

$$
u:=\sup \{v: v \text { is a continuous subsolution to }(1)\},
$$

is a discontinuous solution to (1), i.e., $u^{*}$ is a subsolution and $u=u_{*}$ is a supersolution.

Let $(t, z) \in \Omega_{T}$ be an arbitrary point. By Theorem 1.1, for every $\epsilon>0$, there exists $0<\delta \ll 1$ such that $(t+s, \Phi(s, z)) \in \Omega_{T}$ and

$$
u(t, z)+\epsilon>u^{*}(t+s, \Phi(s, z)) \geq u(t+s, \Phi(s, z)),
$$


for every $|s|<\delta$. Then $\lim \sup _{s \rightarrow 0} u(t+s, \Phi(s, z) \leq u(t, z)+\epsilon$. Letting $\epsilon \searrow 0$, we get $\lim \sup _{s \rightarrow 0} u(t+s, \Phi(s, z) \leq u(t, z)$. Therefore, since $u$ is lower semicontinuous, we have

$$
\lim _{s \rightarrow 0} u(t+s, \Phi(s, z))=u(t, z) .
$$

Moreover, it follows from Theorem 1.1 that for every $\epsilon>0$ there exists $0<\delta \ll 1$ such that $(t+s, \Phi(s, z)) \in \Omega_{T}$ and

$$
u(t+s, \Phi(s, z))+\epsilon>u^{*}(t, z),
$$

for every $|s|<\delta$. Then

$$
\liminf _{s \rightarrow 0} u(t+s, \Phi(s, z))+\epsilon \geq u^{*}(t, z) .
$$

Combining (48) and (49), we have

$$
u(t, z)+\epsilon \geq u^{*}(t, z) .
$$

Letting $\epsilon \searrow 0$, we obtain $u(t, z) \geq u^{*}(t, z)$, and then $u=u_{*}=u^{*}$. Hence, $u$ is a viscosity solution to (1).

Now, we assume that $u_{1}$ and $u_{2}$ are two viscosity solutions to (1). By Theorem 1.1, for every $(t, z) \in \Omega_{T}$ and $\epsilon>0$, there exists $0<\delta \ll 1$ such that $(t+s, \Phi(s, z)) \in \Omega_{T}$ and

$$
u_{1}(t, z)+\epsilon>u_{2}(t+s, \Phi(s, z)),
$$

for every $|s|<\delta$. Since $u_{2}$ is continuous, it implies that

$$
u_{1}(t, z)+\epsilon \geq \lim _{s \rightarrow 0} u_{2}(t+s, \Phi(s, z))=u_{2}(t, z) .
$$

Letting $\epsilon \searrow 0$, we get $u_{1}(t, z) \geq u_{2}(t, z)$. By the same argument, we also have $u_{1}(t, z) \leq$ $u_{2}(t, z)$. Then $u_{1} \equiv u_{2}$.

Thus (1) admits a unique viscosity solution.

\section{ACKNOWLEDGEMENTS}

The second-named author would like to thank Vingroup Innovation Foundation (VINIF) for supporting his Master studies at VNU University of Science, Hanoi.

\section{REFERENCES}

[1] M. G. Crandall, H. Ishii, P. L. Lions, User's guide to viscosity solutions of second order partial differential equations, Bull. Amer. Math. Soc. 27 (1992), no. 1, 1-67.

[2] H-S. Do, G. Le, T. D. Tô, Viscosity solutions to parabolic complex Monge-Ampère equations. Calc. Var. PDEs 59 (2020), no. 2, Paper No. 45, 35 pp.

[3] P. Eyssidieux, V. Guedj, A. Zeriahi, Viscosity solutions to degenerate complex MongeAmpère equations, Comm. Pure Appl. Math. 64 (2011), no. 8, 1059-1094.

[4] P. Eyssidieux, V. Guedj, A. Zeriahi, Weak solutions to degenerate complex MongeAmpère flows I, Math. Ann. 362 (2015), 931-963.

[5] P. Eyssidieux, V. Guedj, A. Zeriahi, Weak solutions to degenerate complex MongeAmpère flows II, Adv. Math. 293 (2016), 37-80.

[6] P. Eyssidieux, V. Guedj, A. Zeriahi, Convergence of weak Kähler-Ricci flows on minimal models of positive Kodaira dimension, Comm. Math. Phys. 357 (2018), no. 3, 1179-1214.

[7] V. Guedj, C.H .Lu, A. Zeriahi, The pluripotential Cauchy-Dirichlet problem for complex Monge-Ampère flows, arXiv:1810.02122 (2018), to appear in Ann.Sc.ENS.

[8] V. Guedj, C.H .Lu, A. Zeriahi, Pluripotential Kähler-Ricci flows, Geom. Topol. 24 (2020), no. $3,1225-1296$. 
[9] V. Guedj, C.H .Lu, A. Zeriahi, Pluripotential solutions versus viscosity solutions to complex Monge-Ampère flows, Pure Appl. Math. Q. 17(2021), no. 3, 971-990.

[10] H. Ishii, On uniqueness and existence of viscosity solutions of fully nonlinear second-order elliptic PDEs, Comm. Pure Appl. Math. 42 (1989), no. 1, 15-45.

[11] H. Ishii, P.L. Lions, Viscosity solutions of fully nonlinear second-order elliptic partial differential equations, Journ. Diff. Equations 83 (1990), 26-78.

[12] R. Jensen: The maximum principle for viscosity solutions of fully nonlinear second-order partial differential equations, Arch. Rat. Mech. Anal. 101 (1988), 1-27.

[13] J. Song, G. Tian, Canonical measures and Kähler Ricci flow, J. Am. Math. Soc. 25, no. 2, 303-353 (2012).

[14] J. Song, G. Tian, The Kähler-Ricci flow through singularities, Invent. Math. 207 (2017), no. 2, 519-595.

[15] T-D. Tô, Convergence of the weak Kähler-Ricci Flow on manifolds of general type, Int. Math. Res. Not. IMRN 2021, No. 8, 6373-6404.

Institute of Mathematics, Vietnam Academy of Science and Technology, 18 Hoang Quoc Viet, Cau Giay, Hanoi, Vietnam

Email address: hoangson.do.vn@gmail.com, dhson@math.ac.vn

Department of Mathematics, VNu University of Science, 334 Nguyen Trai, Thanh Xuan, Ha NoI, VietNam

Email address: phamngocthanhcong1997@gmail.com 